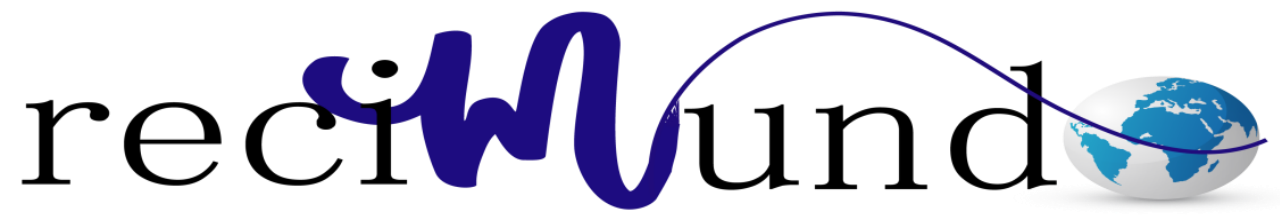

Revista Científica Mundo de la Investigación y el Conocimiento

Irinay Velasco Párraga ${ }^{\text {a }}$; Rocío Suárez Fuentes ${ }^{\text {b }}$; Geny Rivera Salazar ${ }^{\text {c }}$; Herlinda Espinoza Villamar ${ }^{\mathrm{d}}$

Conocimiento sobre el proceso de atención de enfermería del profesional en el cuidado que ofrece al paciente: Unidad de cuidados intensivos cardiovascular. Hospital de niños “Dr. Roberto Gilbert Elizalde”. 2016-2017

Revista Científica Mundo de la Investigación y el Conocimiento. Vol. 2 núm., especial, mayo, ISSN: 2588-073X, 2018, pp. 772-779

DOI: 10.26820/recimundo/2.esp.2018.772-799

Editorial Saberes del Conocimiento

Recibido: 05/12/2017

Aceptado: $15 / 02 / 2018$

a. Hospital de Niños "Dr. Roberto Gilbert Elizalde"

b. Hospital de Niños "Dr. Roberto Gilbert Elizalde"

c. Hospital de Niños "Dr. Roberto Gilbert Elizalde"

d. Hospital de Niños "Dr. Roberto Gilbert Elizalde" 


\section{Conocimiento sobre el proceso de atención de enfermería del profesional en el cuidado que ofrece al paciente: Unidad de cuidados intensivos cardiovascular. Hospital de niños "Dr. Roberto Gilbert Elizalde". 2016-2017}

Vol. 2, núm. Esp., (2018) Irinay Velasco Párraga; Rocío Suárez Fuentes; Geny Rivera Salazar; Herlinda Espinoza Villamar

\section{RESUMEN}

Los Profesionales de Enfermería a nivel Institucional participan activamente en el modelo de calidad de enfermería que se aplica en el hospital, considera como base científica la "teoría de la resonancia del cuidado" siendo sus componentes : interacción humanizada, buena competencia técnica, investigación permanente del cuidado, trabajo en equipo, buena administración de los servicios y seguridad del paciente, en este modelo integra su saber, a una realidad concreta o por evidencia y con la puesta en marcha de metodologías y lenguajes propios (taxonomía). El objetivo de la investigación es determinar el conocimiento sobre el Proceso de Atención de Enfermería del profesional en el cuidado que brinda al paciente en la unidad de Cuidados Intensivos Cardiovascular. Hospital. Dr. Roberto Gilbert Elizalde 2016-2017. Metodología con enfoque cualicuantitativo, descriptivo y prospectivo. Cuantitativo se miden las variables mediante encuestas dirigidas. Cualitativo se utiliza la observación participativa y grupo focal. La muestra es de 20 profesionales de Enfermería. Se considera la variable del Conocimiento sobre el Proceso de Atención de Enfermería y Sub variables: Valoración, Diagnostico, Planificación, Ejecución, Evaluación; los datos demográficos que se correlacionan son: edad, antigüedad en la institución, año de egreso, nivel de formación, tiempo laborando en el servicio. Entre los resultados con respecto al conocimiento en la Valoración 55\%, Diagnostico 62\%, Planificación 50\%, Ejecución 63\%, Evaluación 57\%, y respecto a los datos cualitativos se evidencia que no se aplica el Proceso de Atención de Enfermería y se brinda atención a los pacientes en base a un diagnóstico médico. Se concluye que tienen poco conocimiento, y no se aplica el proceso. Se recomienda cursos de capacitación con referente al Proceso de Atención de Enfermería y el empleo de taxonomía dentro de su desempeño laboral a cabalidad.

Palabras Clave: Proceso de Atención de Enfermería, cuidado, taxonomía. 
Conocimiento sobre el proceso de atención de enfermería del profesional en el cuidado que ofrece al paciente: Unidad de cuidados intensivos cardiovascular. Hospital de niños "Dr. Roberto Gilbert Elizalde". 2016-2017

Vol. 2, núm. Esp., (2018)

Irinay Velasco Párraga; Rocío Suárez Fuentes; Geny Rivera Salazar; Herlinda Espinoza Villamar

\section{ABSTRACT}

Nursing Professionals at the Institutional level actively participate in the nursing quality model applied at the hospital, considered as a scientific basis the "theory of the resonance of care" being its components: humanized interaction, good technical competence, permanent research of the Care, teamwork, good administration of services and patient safety, in this model integrates their knowledge, a concrete reality or by evidence and with the implementation of methodologies and their own languages (taxonomy). The objective of the research is to determine the knowledge about the Nursing Care Process of professional in the care provided to the patient in the Cardiovascular Intensive Care unit. Hospital. Dr. Roberto Gilbert Elizalde 2016-2017. Methodology with a qualitative quanti, descriptive and prospective approach. Quantitative variables are measured through targeted surveys. Qualitative isused forthe participatory observation and focus group are used. The sample is of 20 professionals of Nurses. It is considered the Knowledge variable on the Nursing Care Process and Sub variables: Valuation, Diagnosis, Planning, Execution, Evaluation; The demographic data that correlate are: age, seniority in the institution, year of discharge, level of training, time working in the service. Among the results regarding knowledge in valuation is the $55 \%$, Diagnosis $62 \%$, Planning $50 \%$, Execution 63\%, Evaluation 57\%, and regarding the qualitative data it is evident that the Nursing Care Process is not applied and it is provided Attention to patients based on a medical diagnosis. It is concluded that they have little knowledge, and the process is not applied. Training courses are recommended with regard to the Nursing Care Process and the use of taxonomy within their work performance in full.

Keywords: Nursing care process, care, taxonomy. 


\section{Conocimiento sobre el proceso de atención de enfermería del profesional en el cuidado que ofrece al paciente: Unidad de cuidados intensivos cardiovascular. Hospital de niños "Dr. Roberto Gilbert Elizalde". 2016-2017}

Vol. 2, núm. Esp., (2018) Irinay Velasco Párraga; Rocío Suárez Fuentes; Geny Rivera Salazar; Herlinda Espinoza Villamar

\section{Introducción.}

Aun cuando la enfermería nace como profesión en el siglo XIX con Florence Nigthengel se reconoce la importancia del cuidado, 1955 Lidia Hall describió que las actividades enfermería se determinaban por un proceso y este Proceso de Atención de enfermería se completó con el aporte de algunas teóricas: Roy, Mundinger, Jauron y Aspinall, recién en el año de 1976 añadieron la etapa del diagnóstico, dando lugar a un proceso completo de enfermería quedando definidas las cinco etapas del proceso como lo menciona Alfaro- LR; $2005^{1}$.

Diaz y Nieto; $2011^{2}$, hace referencia que el PAE recién en el año 1977 fue reconocido como "un sistema de intervenciones propias de enfermería sobre la salud de los individuos, familias y comunidades. La Organización Panamericana de la Salud (OPS) en $2007^{3}$ estableció una estrategia regional para el fortalecimiento de la calidad de atención sanitaria y la seguridad del paciente. Estas estrategias se convirtieron en el punto de partida para incorporar obligatoriamente en los planes curriculares de las escuelas y las facultades de enfermería, las bases teóricas elementales acerca del proceso y el empleo inclusive de las taxonomías NANDA, NIC y NOC (NNN), como un lenguaje enfermero y otras tecnologías didácticas para estandarizar los cuidados, así como su aplicación en la práctica como lo menciona Rojas JG; 20074 . Esto permitió emplear las pautas que delimita la teoría y evita discrepancias en su aplicación entre un profesional y otro, además de estandarizar el lenguaje mediante el uso exclusivo del proceso.

A pesar de todos los esfuerzos por la aplicación del PAE, su incorporación a la práctica de enfermería ha sido muy lenta y heterogénea; y el personal de enfermería de las áreas de 
Conocimiento sobre el proceso de atención de enfermería del profesional en el cuidado que ofrece al paciente: Unidad de cuidados intensivos cardiovascular. Hospital de niños "Dr. Roberto Gilbert Elizalde". 2016-2017

Vol. 2, núm. Esp., (2018)

Irinay Velasco Párraga; Rocío Suárez Fuentes; Geny Rivera Salazar; Herlinda Espinoza Villamar

hospitalización aún expresa deficiencias en su comprensión, manejo e implementación. Su falta de aplicación pudiera deberse a factores como los pocos conocimientos que hay sobre el tema, de acuerdo a Corzo RM, Montes ZM, Ramírez MS, Reyes RL, Rodríguez HA; $2004^{5}$ y Chávez T; $2006^{6}$ o bien a la resistencia a aplicarlo por parte del personal, como lo menciona en el Programa Nacional de Salud (2007-2012). Si el problema en la aplicación de este proceso prevaleciera, quizá la disciplina nunca alcanzaría el punto máximo de su profesionalización y nunca se lograría un lenguaje unificado, ya que faltarían las bases científicas en la práctica que fundamentaran los cuidados brindados, no serían estandarizados y se dudaría de su calidad.

En Canadá las enfermeras presentaban problemas para construir un diagnóstico y el $44 \%$ de las profesionales no se basaban en los factores etiológicos ${ }^{7}$. Es importante para poder llegar a un diagnóstico de calidad y uniformidad en las intervenciones y resultados de enfermería, ampliar el conocimiento de varias taxonomías, para implementarlo en la planificación de los cuidados de enfermería ${ }^{8}$. En un estudio realizado en Suecia desde el punto de vista cualitativo los resultados de las intervenciones de enfermería documentados mejoraron significativamente posterior a un adiestramiento. Bjoervell et al.; $2002^{9}$ al mismo tiempo mejoró la calidad de la documentación y aumentó la satisfacción del paciente en un hospital de los Estados Unidos Nahm R, Poston I; $2000^{10}$ luego de la intervención de la unificación de la documentación.

En España existen planes de cuidados normalizados y unificados a través del proyecto NIPE, es indispensable utilizar lenguajes unificados y metodología homogénea con apoyo de herramientas informáticas para el registro y documentación de los cuidados de enfermería ${ }^{11}$. Como resultado de lo anteriormente expresado, el proceso de Atención de Enfermería a nivel 


\section{Conocimiento sobre el proceso de atención de enfermería del profesional en el cuidado que ofrece al paciente: Unidad de cuidados intensivos cardiovascular. Hospital de niños "Dr. Roberto Gilbert Elizalde". 2016-2017}

Vol. 2, núm. Esp., (2018) Irinay Velasco Párraga; Rocío Suárez Fuentes; Geny Rivera Salazar; Herlinda Espinoza Villamar latinoamericano y mundial comenzó a formar parte de la malla curricular de las Universidades en los programas de enfermería, porque reconocen que es un instrumento necesario que ofrece directrices lógicas, secuencial y evaluable para satisfacer las necesidades del cuidado de los sujetos a nivel asistencial y comunitario.

En una Universidad Nacional de Colombia Díaz y Nieto; $1998^{12}$ se realizó un estudio en seis instituciones de salud en Bogotá donde se demostró que el proceso no es bien definido y no se lo usa mucho debido a que el aprendizaje lo recibieron hace mucho tiempo, poca actualización y no se tiene claro las metas de cuidado en la práctica asistencial, esto es confirmado por otro estudio realizado en Villavicencio/Colombia por Gutiérrez; $2008^{13}$ en cuatro instituciones donde se evidenció que el proceso era aplicado muy poco debido a: falta de tiempo, dedicación a la administración de medicamentos, el tiempo de ejercicio profesional y conocimiento deficiente sobre el proceso. Así también se evidencia poco juicio crítico sobre el proceso de saludenfermedad de los sujetos de cuidado por lo que es muy poco utilizado en las instituciones de salud.

Aun cuando la utilización del proceso de Atención de enfermería es escasa en las entidades de salud, como por ejemplo el Hospital Roberto Gilbert Elizalde se plantea indagar sobre el conocimiento y la práctica del PAE en la atención de los pacientes, por lo tanto los resultados de las investigaciones previas permitirán comparar cual es nuestra realidad en comparación a otros países. 
Conocimiento sobre el proceso de atención de enfermería del profesional en el cuidado que ofrece al paciente: Unidad de cuidados intensivos cardiovascular. Hospital de niños "Dr. Roberto Gilbert Elizalde". 2016-2017

Vol. 2, núm. Esp., (2018)

Irinay Velasco Párraga; Rocío Suárez Fuentes; Geny Rivera Salazar; Herlinda Espinoza Villamar

Siendo así, el Ecuador, no está lejos de esta realidad ya que en la ciudad de Cuenca existe un trabajo de investigación titulado "Aplicación del proceso de atención de enfermería de las enfermeras/os, que laboran en el Hospital Regional Vicente Corral Moscoso, Cuenca 2015". Vele y Veletanga; $2015^{14}$. En el cual de acuerdo a los resultados se concluyó que el P.A.E no se lo aplica adecuadamente y al momento de brindar el cuidado a los usuarios aplican otras metodologías, el 65\% desconocen el proceso del PAE aunque lo consideran importante y que ayuda a mantener la calidad de atención brindada por el profesional de enfermería.

Considerando la información del estudio anteriormente expuesto es necesario hacerse la pregunta : ¿cuál es el conocimiento y practica del PAE de los profesionales de enfermería en el hospital de niños "Dr. Roberto Gilbert Elizalde" de la ciudad de Guayaquil?. El Hospital es una institución regentada por la Junta de Beneficencia de Guayaquil, tiene 128 años al servicio de la comunidad, atiende a niños desde 0 días a 17 años 11 meses 29 días de edad. Es de complejidad de tercer nivel y atiende a pacientes a nivel, teniendo más afluencias de las provincias de: Manta, Los Ríos, El Oro; con una capacidad de 460 camas; oferta servicios de atención: Consulta externa, Laboratorio Clínico, Imágenes, Emergencia, Hospitalización como las siguientes áreas: general servicios de electrodiagnósticos, Cirugía, cuidados intensivos de neonatos, pediátrica y cardiológica, quemados.

La unidad de Cuidados Intensivos Cardiovascular, con capacidad para 12 pacientes, atiende a neonatos con peso mayor de $2.5 \mathrm{~kg}$, lactante menor y mayor, pediátricos, que requieren tratamiento quirúrgico o que sean sometidos a cateterismos cardiacos con finalidad diagnóstico o terapéutico, también se brinda atención de adultos con cardiopatías congénitas no corregidas a la 


\section{Conocimiento sobre el proceso de atención de enfermería del profesional en el cuidado que ofrece al paciente: Unidad de cuidados intensivos cardiovascular. Hospital de niños "Dr. Roberto Gilbert Elizalde". 2016-2017}

Vol. 2, núm. Esp., (2018) Irinay Velasco Párraga; Rocío Suárez Fuentes; Geny Rivera Salazar; Herlinda Espinoza Villamar edad correspondientes, los que son derivados del hospital Luis Vernaza. El talento humano tiene una praxis profesional en la especialidad en la atención de pacientes con alta complejidad, de esta forma se ofrece un cuidado de enfermería y médico en forma individualizada y de alta calidad.

La dotación del personal en el servicio es de: 6 médicos tratantes y 3 residentes distribuidos en diferentes turnos; personal de enfermería: 18 profesionales y 9 auxiliares distribuidas de las siguiente manera: 4 profesionales por cada turno de la mañana y tarde, y 3 en la noche. Auxiliares 2 por turno. El profesional de enfermería administra el cuidado directo al paciente a un máximo de 3 pacientes $\mathrm{x}$ turno, brindando cuidados como: toma de signos vitales, tratamientos especializados, administración de medicamentos. El personal de auxiliar de enfermería brinda atención exclusiva de higiene y confort al paciente.

Para garantizar una atención de enfermería de calidad, es necesario que tenga un pensamiento unificado acerca de su significado y esencia que es el cuidado, todo ello evidenciado en la definición de un modelo de calidad de cuidado, cuyos componentes se correspondan con la esencia del cuidado de enfermería y las teorías de calidad.

El modelo de calidad de enfermería que se aplica en el hospital, considera como base científica la "teoría de la resonancia del cuidado" siendo sus componentes 6: interacción humanizada, buena competencia técnica, investigación permanente del cuidado, trabajo en equipo, buena administración de los servicios y seguridad del paciente. La implementación del modelo de calidad se hace utilizando estrategia del modelo de educación permanente, basada en 
Conocimiento sobre el proceso de atención de enfermería del profesional en el cuidado que ofrece al paciente: Unidad de cuidados intensivos cardiovascular. Hospital de niños "Dr. Roberto Gilbert Elizalde". 2016-2017

Vol. 2, núm. Esp., (2018)

Irinay Velasco Párraga; Rocío Suárez Fuentes; Geny Rivera Salazar; Herlinda Espinoza Villamar

el modelo pedagógico de la problematización de Paulo Freire, utilizando como unidad de análisis

"la problematización o análisis crítico de la práctica cotidiana basado en método científico. El presente modelo se lo adapta para lograr la actualización permanente de la práctica y su adaptación a los cambios del entorno sociopolítico, económico y cultural, el departamento de enfermería a considerado necesario construir en colaboración de todos sus miembros un modelo de calidad del cuidado que guie hacia la consecución de un primer nivel de calidad.

Con el modelo anteriormente mencionado, en el que el /la enfermero(a) integra su saber, a una realidad concreta o por evidencia. Se va a conocer la praxis que ha durante la atención del paciente de los componentes de la buena competencia técnica e investigación permanente del cuidado, en donde se identifican como criterios de aplicación y evaluación de las etapas del proceso de atención enfermero, permitiendo así estandarizar y aplicar el lenguaje propio del profesional de enfermería.

Este actuar implica buscar datos, analizarlos, diagnosticarlos, que va a dar lugar a las intervenciones para posterior evaluación, cuyos resultados permitirán iniciar el proceso aplicando la teoría de sistema, además permite obtener base de datos para realizar Investigaciones por evidencia o Clínica, mantener una identidad profesional y una atención unificada, brindando atención humanizada, holística y organizada para realizar el plan de cuidado con la aplicación del método de enfermería. 


\section{Conocimiento sobre el proceso de atención de enfermería del profesional en el}

cuidado que ofrece al paciente: Unidad de cuidados intensivos cardiovascular. Hospital de niños "Dr. Roberto Gilbert Elizalde". 2016-2017

Vol. 2, núm. Esp., (2018) Irinay Velasco Párraga; Rocío Suárez Fuentes; Geny Rivera Salazar; Herlinda Espinoza Villamar

El objetivo de la presente investigación busca determinar el conocimiento sobre el proceso de atención de enfermería del profesional en el cuidado que brinda al paciente en la unidad de cuidados intensivos cardiológico. Hospital. Dr. Roberto Gilbert Elizalde 2016-2017.

\section{Metodología.}

\section{Diseño metodológico}

Al realizar el análisis de la problematización que se presenta en los profesionales relacionado con la aplicación del proceso de atención de enfermería en la unidad de cuidados intensivos cardiovascular, es necesario realizar una investigación-acción con la finalidad de dar solución al problema planteado. Siendo así, el presente trabajo tiene tres etapas:

1. Diagnóstica, que concluye con la propuesta de solución al problema.

2. Implementación de la propuesta.

3. Control y Evaluación de la aplicación de la propuesta. Estudio comparativo.

Para la primera etapa que es la Diagnóstica, se realizó un estudio con un enfoque de diseño cualicuantitativo, los métodos que se aplicaron son: inductivo-deductivo, histórico lógico y de triangulación. El tipo de la investigación fue descriptivo, prospectivo e investigación acción.

Lo que corresponde al diseño cuantitativo, donde se cuantificaron las variables mediante la aplicación de la encuesta y cualitativo en el que se analizó la percepción de la aplicación del 
Conocimiento sobre el proceso de atención de enfermería del profesional en el cuidado que ofrece al paciente: Unidad de cuidados intensivos cardiovascular. Hospital de niños "Dr. Roberto Gilbert Elizalde". 2016-2017

Vol. 2, núm. Esp., (2018)

Irinay Velasco Párraga; Rocío Suárez Fuentes; Geny Rivera Salazar; Herlinda Espinoza Villamar

Proceso de Atención de Enfermería por el personal profesional en el área de Cuidados Intensivos Cardiovascular con la técnica de grupo focal y la observación participativa.

El tipo de investigación que se aplicó fue descriptivo porque permitió hacer un diagnóstico para conocer la situación actual y medir las dimensiones del proceso. Es prospectivo por qué se midió la aplicación del conocimiento actual del Proceso de Atención de Enfermería (PAE) en todos los profesionales de enfermería del área de cuidados intensivos cardiovascular del hospital Dr. Roberto Gilbert Elizalde desde septiembre a diciembre del año 2016, y las siguientes etapas se cumplirán en el 2017, por esta razón es considero una investigación - acción.

\section{Población y muestra}

La Población de los profesionales de enfermería que laboran en el Hospital de niños Dr. Roberto Gilbert Elizalde es de 319. El trabajo se realizó en el área de Cuidados Intensivos Cardiovascular en el que hay 20 profesionales considerándolo como la muestra para la ejecución de la investigación, siendo así es una muestra no probabilística - condicionada, por lo que los criterios de inclusión son: profesionales de enfermería que tienen relación de dependencia en la institución y que laboren en el área de cuidados intensivos cardiovascular a quienes se les aplicó la encuesta cuantitativa.

Para la investigación cualitativa de los grupos focales se tomó una muestra de 6 profesionales del área y la se aplicó una guía de observación participativa a los 20 profesionales. 


\section{Conocimiento sobre el proceso de atención de enfermería del profesional en el cuidado que ofrece al paciente: Unidad de cuidados intensivos cardiovascular. Hospital de niños "Dr. Roberto Gilbert Elizalde". 2016-2017}

Vol. 2, núm. Esp., (2018) Irinay Velasco Párraga; Rocío Suárez Fuentes; Geny Rivera Salazar; Herlinda Espinoza Villamar

\section{Recolección de Datos}

En la recolección de los datos cuantitativo, se aplicó una encuesta al personal de enfermería con preguntas cerradas, dirigidas por las variables de estudio para obtener datos sobre el conocimiento del proceso de atención de enfermería en la atención que brinda a los pacientes. Se comprobó la factibilidad y validez de la encuesta a través de una prueba piloto con 5 participantes, cuyo resultado confirmó su aplicación.

Para la recolección de los datos cualitativo se aplicó la técnica de observación participativa a los profesionales en Enfermería durante el desarrollo de su trabajo, para lo cual se diseñó una guía de observación y dichos datos se complementan con la técnica de Grupo focal donde se estructuraron 5 preguntas directrices con la finalidad de obtener la información, lo que permitió realizar la triangulación de datos.

\section{Procesamiento de Datos}

Para la investigación cuantitativa (Encuesta de selección múltiple) para el procesamiento de datos se utilizó el sistema informático de Microsoft Excel en el que se estructuró la base de datos que permitió realizar las tablas estadísticas y los gráficos.

Los datos obtenidos en la investigación cualitativa (Observación participativa y grupo focal), para el procesamiento se utilizaron el sistema informático de Microsoft Excel para correlacionar los datos cuantitativos y cualitativos y Word para narrar la información obtenida del audio, lo que permitió realizar la categorización y la determinación de los núcleos de sentido. 
Conocimiento sobre el proceso de atención de enfermería del profesional en el cuidado que ofrece al paciente: Unidad de cuidados intensivos cardiovascular. Hospital de niños "Dr. Roberto Gilbert Elizalde". 2016-2017

Vol. 2, núm. Esp., (2018)

Irinay Velasco Párraga; Rocío Suárez Fuentes; Geny Rivera Salazar; Herlinda Espinoza Villamar

Análisis e interpretación de la información

En la investigación cuantitativa el análisis e interpretación de los datos se realizó en base a la correlación entre la variable proceso de atención de enfermería y las subvariables que son la aplicación de cada uno de las etapas, para evidenciar de esta manera el conocimiento del profesional de enfermería y así plantear la propuesta educativa como solución al problema encontrado.

La investigación cualitativa se lo realizó a través del análisis de contenidos, este se correlacionó con las teorías seleccionadas para el estudio. De esta forma se realizó un análisis a profundidad de la problemática aplicando el método de la triangulación.

Según Denzin (1970) "la triangulación es la combinación de dos o más teorías fuentes de datos, métodos de investigación en el estudio de un fenómeno singular”. El tipo de triangulación que se utilizó fue de datos, en el que se confrontan los cuantitativos y cualitativos en concordancia con las teorías seleccionadas.

\section{Consideraciones Éticas}

Para su ejecución, esta investigación contó con la autorización y seguimiento de la dirección técnica y el comité de ética del hospital; además de la respectiva firma de consentimiento informado de todos los participantes.

Para garantizar la confidencialidad de estos datos, la información obtenida se la entregó al Departamento de Docencia del Hospital para su respectivo archivo. 


\section{Conocimiento sobre el proceso de atención de enfermería del profesional en el}

cuidado que ofrece al paciente: Unidad de cuidados intensivos cardiovascular. Hospital de niños "Dr. Roberto Gilbert Elizalde". 2016-2017

Vol. 2, núm. Esp., (2018) Irinay Velasco Párraga; Rocío Suárez Fuentes; Geny Rivera Salazar; Herlinda Espinoza Villamar

\section{Resultados.}

Análisis de las etapas del PAE

Una vez obtenidos los datos se procede a la tabulación, luego se realiza el análisis e interpretación de los datos en donde se evalúa el "Conocimiento sobre el Proceso de Atención de Enfermería del profesional en el cuidado que ofrece al paciente". En esta investigación se ha considerado las etapas del Proceso de Atención de Enfermería avalado por la Organización Mundial de la Salud para relacionarlos con los resultados. Esta metodología es el producto de un modelo que nos permite comparar la evidencia científica con la práctica actual, los profesionales de enfermería del Hospital Roberto Gilbert es de 360. Se realizó en el área de Cuidados Intensivos Cardiovascular, en la que laboran 20 Licenciadas/os en Enfermería los que se consideraron como la población objeto de estudio.

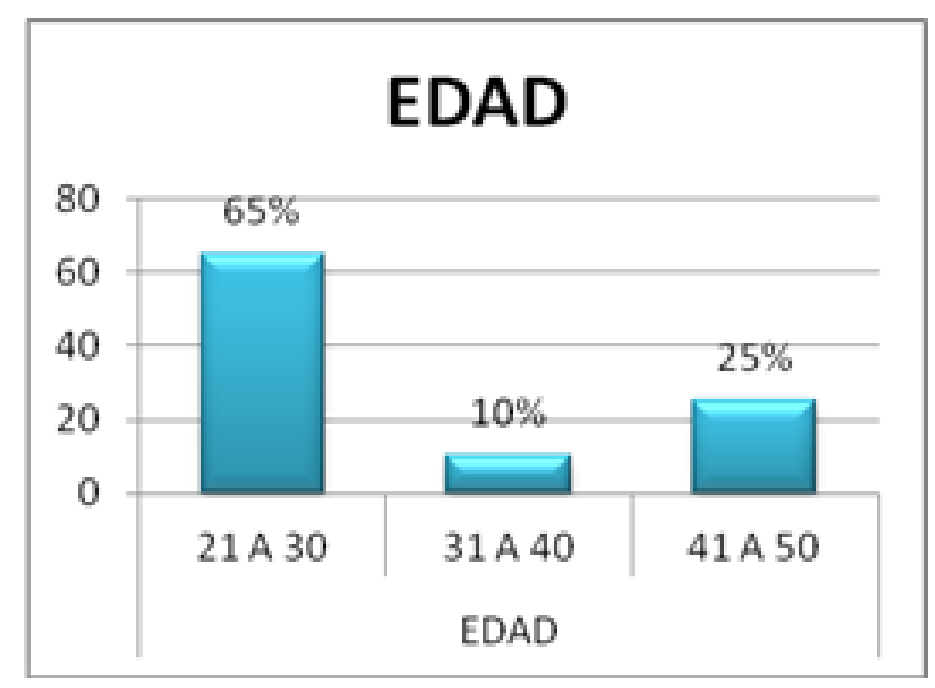

Grafico $N^{\circ}$ 1.- Edad 
Conocimiento sobre el proceso de atención de enfermería del profesional en el cuidado que ofrece al paciente: Unidad de cuidados intensivos cardiovascular. Hospital de niños "Dr. Roberto Gilbert Elizalde". 2016-2017

Vol. 2, núm. Esp., (2018)

Irinay Velasco Párraga; Rocío Suárez Fuentes; Geny Rivera Salazar; Herlinda Espinoza Villamar

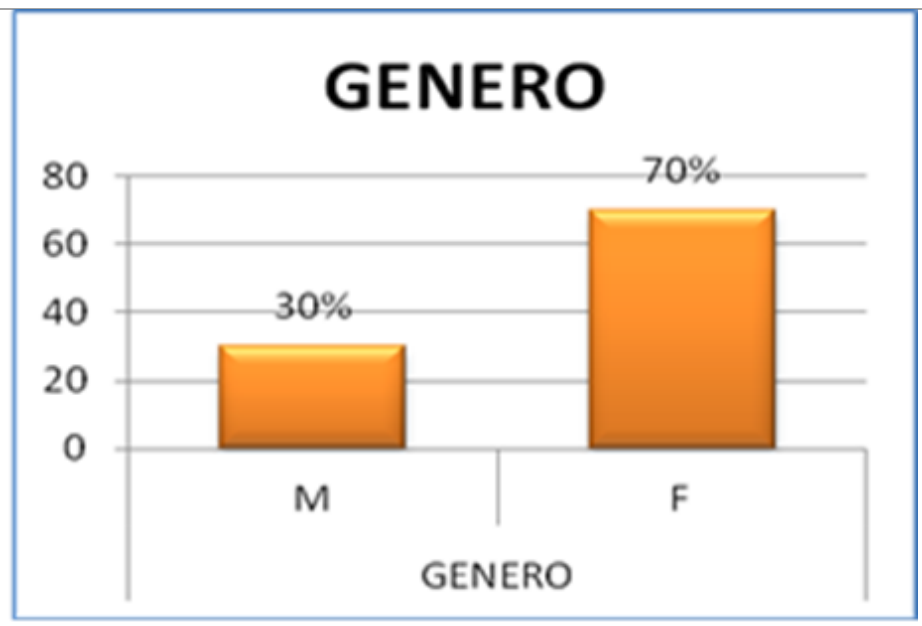

Grafico $N^{\circ}$ 2.- Genero

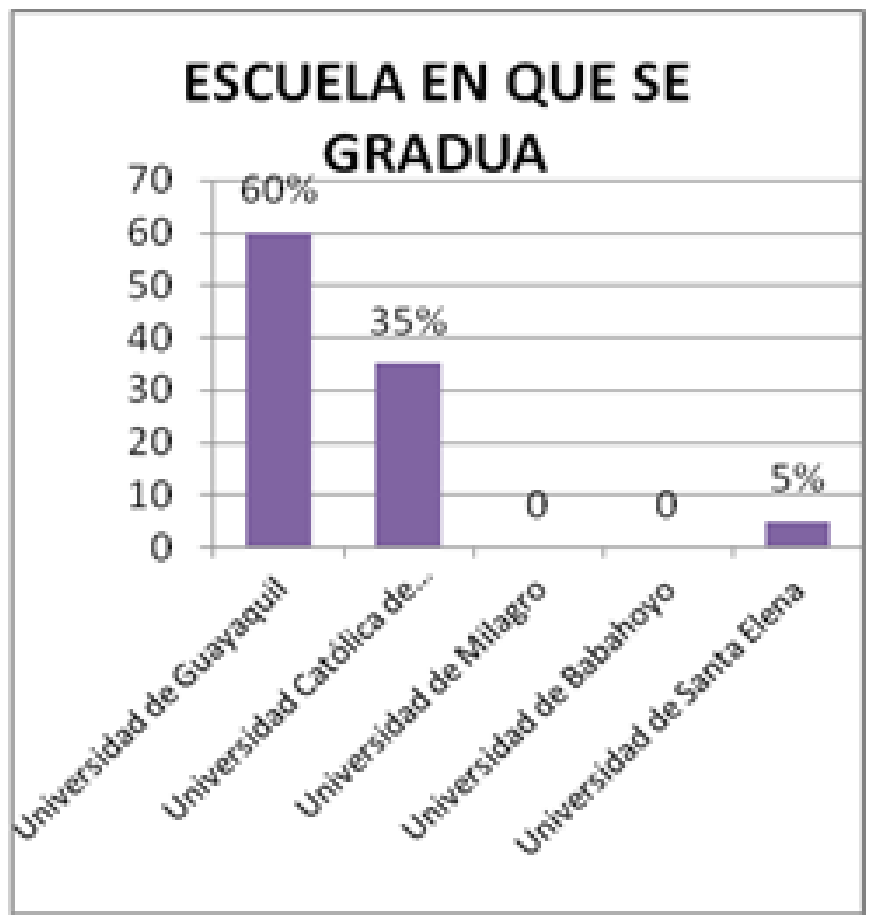

Grafico $N^{\circ}$ 3.- Universidades 
Conocimiento sobre el proceso de atención de enfermería del profesional en el cuidado que ofrece al paciente: Unidad de cuidados intensivos cardiovascular. Hospital de niños "Dr. Roberto Gilbert Elizalde". 2016-2017

Vol. 2, núm. Esp., (2018) Irinay Velasco Párraga; Rocío Suárez Fuentes; Geny Rivera Salazar; Herlinda Espinoza Villamar

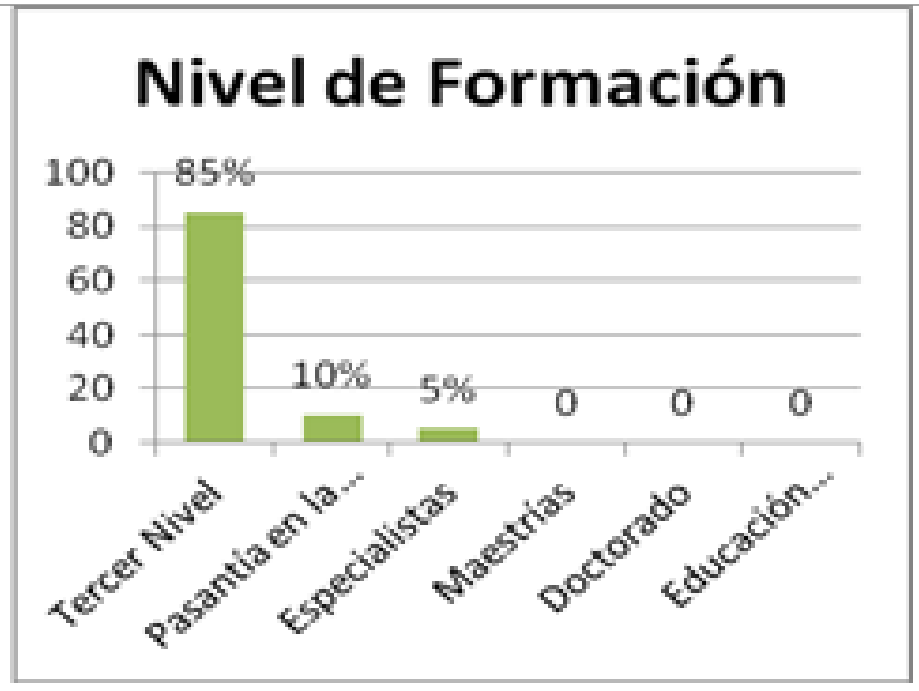

Grafico $N^{\circ}$ 4.- Nivel de Formación

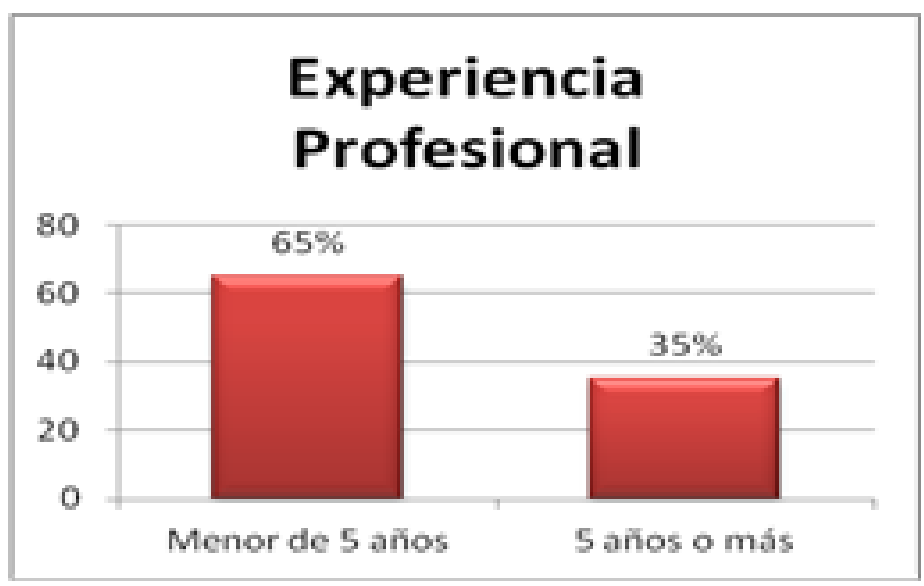

Grafico $N^{\circ}$ 5.- Experiencia 
Conocimiento sobre el proceso de atención de enfermería del profesional en el cuidado que ofrece al paciente: Unidad de cuidados intensivos cardiovascular. Hospital de niños "Dr. Roberto Gilbert Elizalde". 2016-2017

Vol. 2, núm. Esp., (2018)

Irinay Velasco Párraga; Rocío Suárez Fuentes; Geny Rivera Salazar; Herlinda Espinoza Villamar

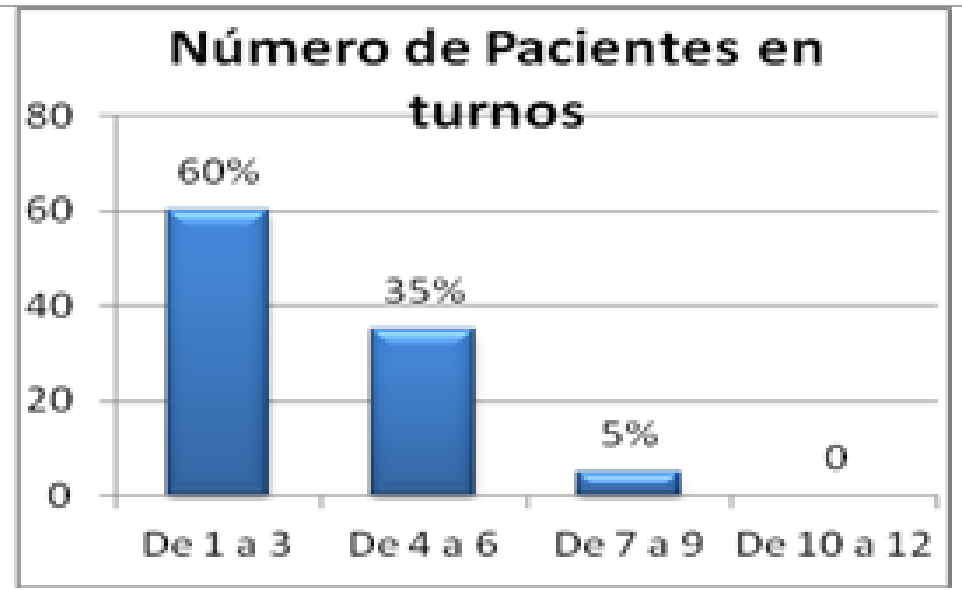

Grafico $N^{\circ}$ 6.- Turnos

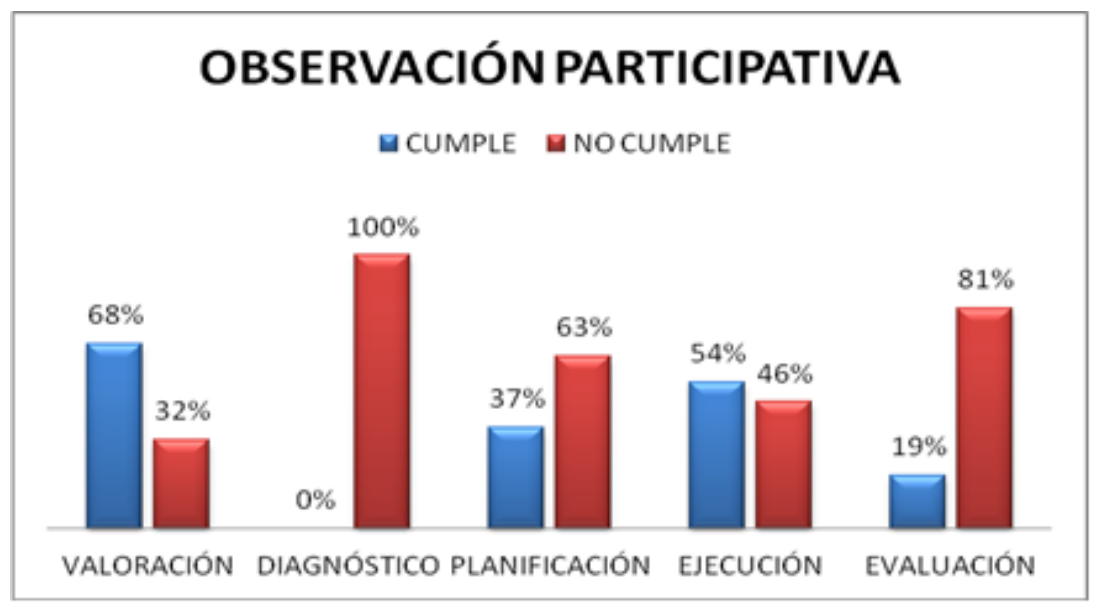

Grafico $N^{\circ}$ 7.- Observación 


\section{Conocimiento sobre el proceso de atención de enfermería del profesional en el}

cuidado que ofrece al paciente: Unidad de cuidados intensivos cardiovascular. Hospital de niños "Dr. Roberto Gilbert Elizalde". 2016-2017

Vol. 2, núm. Esp., (2018) Irinay Velasco Párraga; Rocío Suárez Fuentes; Geny Rivera Salazar; Herlinda Espinoza Villamar

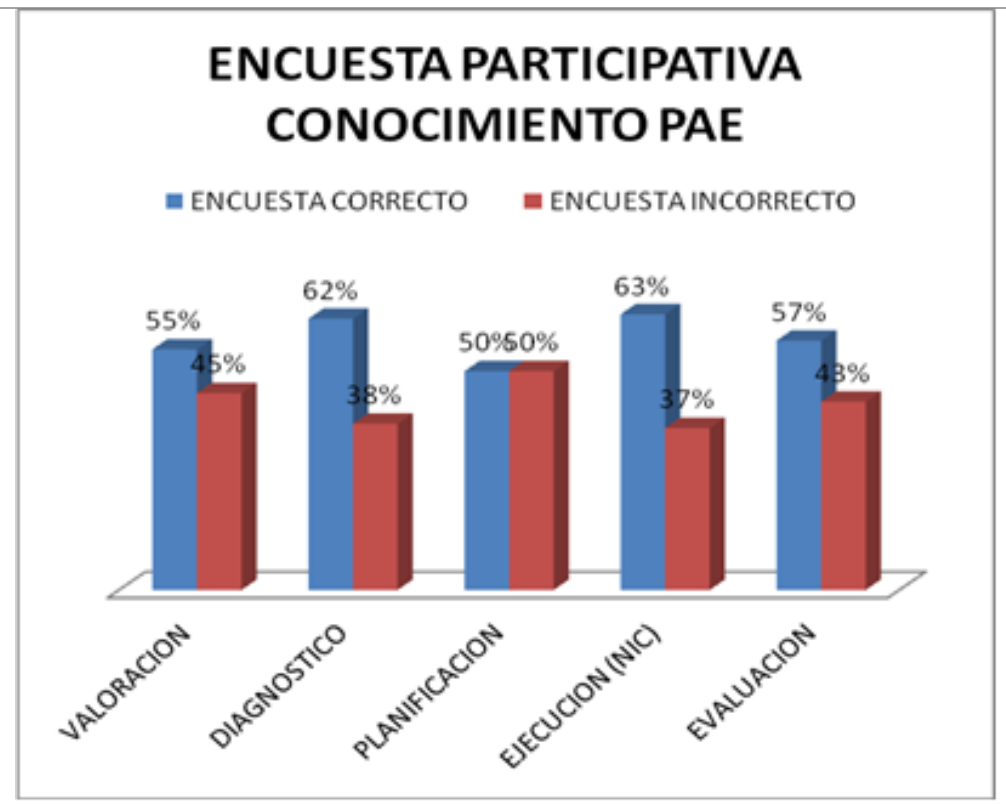

Grafico $N^{\circ}$ 8.- Encuesta

El $70 \%$ son mujeres y un $30 \%$ son hombres, de los cuales el $60 \%$ son graduados en la Universidad de Guayaquil y el $40 \%$ en la Universidad Católica de Guayaquil, el 75\% son adultos jóvenes (18-30 años) según la OMS, y el 25\% de los profesionales de edad madura (31-45 años) según OMS; el 85\% tiene una formación de tercer nivel, el 10\% a realizado pasantías en el cuidado de pacientes post quirúrgicos cardiacos en países como Chile y México solo un 5\% tiene la especialidad en enfermería pediátrica, el 65\% tiene experiencia laboral entre uno y dos años en el servicio y un 35\% tiene entre 6 y 10 años laborando en el servicio; con respecto al parámetro de medición del número de pacientes por profesional; el $60 \%$ de los encuestados manifiesta que tiene un numero apropiado de pacientes por profesional (1-3 pacientes); el 35\% manifiesta que tiene una cantidad intermedia de pacientes (4-6 pacientes); y solo un 5\% manifiesta que tiene un exceso de paciente (7-10 pacientes). De acuerdo a lo que dice 
Conocimiento sobre el proceso de atención de enfermería del profesional en el cuidado que ofrece al paciente: Unidad de cuidados intensivos cardiovascular. Hospital de niños "Dr. Roberto Gilbert Elizalde". 2016-2017

Vol. 2, núm. Esp., (2018)

Irinay Velasco Párraga; Rocío Suárez Fuentes; Geny Rivera Salazar; Herlinda Espinoza Villamar

Baldera 2015 en las áreas de cuidados intensivos debería de haber 100\% de profesionales con tres profesionales necesarios para suplencias, distribuidos de la siguiente manera un $40 \%$ matutino y un $30 \%$ vespertino y nocturno, eso significa que en nuestro servicio deberíamos de tener 17 profesionales, aparte los tres profesionales suplentes. Esto nos confirma que contamos con un número adecuado de personal para la atención de nuestros pacientes.

\section{Valoración}

En el Primer objetivo de la investigación sobre el conocimiento de la etapa de valoración del Proceso de Atención de Enfermería en relación a la encuesta dirigida se evidencia que un promedio, el 55\% de los profesionales en enfermería tienen conocimiento científico, siendo lo más relevante de las 15 preguntas aplicadas: el concepto (85\%), los tipos de recogida de datos (85\%), los métodos de recolección de datos (80\%) y las técnicas de exploración (90\%), pero se debe fortalecer el conocimiento en: fuentes de datos primarias (25\%), las partes de observación (15\%), la síntesis de la información (25\%) y las fases de la valoración (20\%). Mientras que, en el análisis de la observación participativa el 68\% de los profesionales en enfermería ejecutan con base científica los componentes de la atapa de valoración, siendo lo más relevante de las 7 enunciados de la guía de observación participativa: criterios de valoración, técnicas de exploración, examen físico teniendo que fortalecer: síntesis de de información; y con respecto al grupo focal se obtuvo como núcleo de sentido que "la valoración se realiza de manera desordenada" lo que se corrobora con expresiones de los participantes como:

PARTICIPANTE 1 "Entrevistamos, Auscultamos, Palpamos, Priorizamos". 


\section{Conocimiento sobre el proceso de atención de enfermería del profesional en el cuidado que ofrece al paciente: Unidad de cuidados intensivos cardiovascular. Hospital de niños "Dr. Roberto Gilbert Elizalde". 2016-2017}

Vol. 2, núm. Esp., (2018) Irinay Velasco Párraga; Rocío Suárez Fuentes; Geny Rivera Salazar; Herlinda Espinoza Villamar PARTICIPANTE 2 "Entrevista con el paciente, anamnesis, examen físico".

En base a los resultados de la triangulación coinciden en que profesionales de enfermería tienen conocimiento científico básico, y lo que se aplica en la práctica es desordenado e incompleto. Siendo así, que la valoración es la primera fase del proceso donde el profesional planea sus actividades de forma continua. Como lo afirma Phaneuf, que la valoración es un proceso organizado, deliberado y sistemático de recolección de información pertinente sobre el usuario, la familia o comunidad que requiere la atención; con el propósito de identificar datos relevantes para determinar los problemas, necesidades, respuestas humanas y riesgos.

\section{Diagnóstico}

En el segundo objetivo de la investigación sobre la obtención del Diagnóstico en base a la Valoración del paciente en relación a la encuesta dirigida se evidencia que un $62 \%$ de los profesionales de enfermería tienen conocimiento científico, siendo la más relevante de las 6 preguntas aplicadas: estructura de un diagnostico (100\%) pero se debe fortalecer: niveles de la taxonomía del diagnóstico (40\%). Mientras que en el análisis que contiene la observación el $50 \%$ de los profesionales de enfermería ejecutan con base científica los componentes de la etapa del diagnóstico, siendo lo más relevante de los 6 enunciados de la guía de observación participativa: a través de que se estructura un diagnostico teniendo que fortalecer componentes para el enunciado del diagnóstico, y con respecto al grupo focal se obtuvo como núcleo de sentido que "el Diagnóstico lo realizan participantes de forma desordenada, y no tenían 
Conocimiento sobre el proceso de atención de enfermería del profesional en el cuidado que ofrece al paciente: Unidad de cuidados intensivos cardiovascular. Hospital de niños "Dr. Roberto Gilbert Elizalde". 2016-2017

Vol. 2, núm. Esp., (2018)

Irinay Velasco Párraga; Rocío Suárez Fuentes; Geny Rivera Salazar; Herlinda Espinoza Villamar

sentido las respuestas que daban" lo que se corrobora con expresiones de los participantes como:

1: "Planificación, Planificamos, Patrones funcionales, Diagnósticos, Intervenciones".

PARTICIPANTE 3: "Intervenciones, Diagnóstico, Planificas, Ejecutamos, Patrones funcionales, computadora".

PARTICIPANTE 5: "No me acuerdo".

En base a los resultados de la triangulación coinciden en que profesionales de enfermería solo conocen en base a que estructura se obtiene un Diagnostico, el mismo que se conoce pero no se evidencia su aplicación en la práctica, esto significa que el cuidado de enfermería se lo está realizando de acuerdo a un diagnóstico médico, el diagnóstico no tiene que plantarse como una etapa terminal o como fin del proceso de cuidar en enfermería, sino que es el resultado del proceso de valorar el punto de arranque para la planificación y lo subsiguiente .Los diagnósticos son solo etiquetas que identifican y nombran las situaciones de cuidar, es decir, expresan una conclusión.

PLANIFICACIÓN (Resultados e intervención)

El tercer objetivo de la investigación sobre la estructura los resultados y aplicación las intervenciones en la atención del paciente en relación a la encuesta dirigida se evidencia que un 57\% de los profesionales de enfermería tiene conocimiento científico, siendo las más relevantes de las 17 preguntas aplicadas: Concepto de planificación (90\%), recomendaciones para realizar 


\section{Conocimiento sobre el proceso de atención de enfermería del profesional en el cuidado que ofrece al paciente: Unidad de cuidados intensivos cardiovascular. Hospital de niños "Dr. Roberto Gilbert Elizalde". 2016-2017}

Vol. 2, núm. Esp., (2018) Irinay Velasco Párraga; Rocío Suárez Fuentes; Geny Rivera Salazar; Herlinda Espinoza Villamar el registro $(90 \%)$, concepto de ejecución(85\%), tipos de intervenciones $(85 \%)$, fases de ejecución(80\%) pero se debe fortalecer objetivos de la planificación (20\%), elementos de la intervención (20\%) y características de la planificación(15\%). Mientras que en el análisis que contiene la observación el $45 \%$ de los profesionales de enfermería ejecutan con base científica los componentes de la etapa de la Planificación, siendo lo más relevante de los 11 enunciados de la guía de observación participativa: las características de la redacción de las intervenciones (100\%) y los elementos de la intervención(89\%), objetivos de la ejecución(84\%), características de la planificación(84\%), recomendaciones para realizar el registro (89\%), teniendo que fortalecer: Objetivos de la planificación, fases de la planificación, componentes de los objetivos, fases de la ejecución (5\%) y actividades durante la fase preparación (0\%), y con respecto al grupo focal se obtuvo como núcleo de sentido que conocen que "Hay que estructurar resultados pero lo hacen de forma desorganizada sin tener evidencia del cuidado y las intervenciones que se le brinda al paciente son en base al diagnóstico médico" lo que se corrobora con expresiones de los participantes como:

PARTICIPANTE 4: "Un aspecto importante de la planificación es también la descripción de las tareas necesarias para dar una respuesta individualizada a la situación de cada sujeto".

PARTICIPANTE 4: "Los diagnostico con los exámenes así evidenciamos, registramos en la hoja de balance así evidenciamos que hemos trabajado".

PARTICIPANTE 3: "Hacemos la intervención y esperamos el tiempo necesario para poder reevaluar". 
Conocimiento sobre el proceso de atención de enfermería del profesional en el cuidado que ofrece al paciente: Unidad de cuidados intensivos cardiovascular. Hospital de niños "Dr. Roberto Gilbert Elizalde". 2016-2017

Vol. 2, núm. Esp., (2018)

Irinay Velasco Párraga; Rocío Suárez Fuentes; Geny Rivera Salazar; Herlinda Espinoza Villamar

En base a los resultados de la triangulación coinciden en que los profesionales de enfermería solo conocen que cuando se elabora la etapa de planificación, se tendrá que hacer referencia a los protocolos de actuación a los llamados G.D.R (Grupos de Diagnósticos Relacionados) 44, a los proyectos N.I.C (Nursing Intervetions Classification) y N.O.C (Nursing Outcomes Classification). La enfermera/o necesita de la habilidad manual, la capacidad técnica, así como de sus capacidades intelectuales, de interrelaciones personales, toma de decisiones, observación y comunicación para así garantizar el éxito de su actuación.

\section{Evaluación}

En el cuarto objetivo sobre la verificación el registro de la evaluación de las intervenciones en el cuidado del paciente en relación a la encuesta dirigida se evidencia que un promedio $57 \%$ de los profesionales en enfermería tienen conocimiento científico, siendo lo más relevantes de las 6 preguntas aplicadas: la conceptualización de evaluación (85\%) y los objetivos de la evaluación (80\%) pero se debe fortalecer el conocimiento en: los pasos de la evaluación de acuerdo al NANDA (10\%). Mientras que, en el análisis de la observación participativa solo el 19\% de los profesionales en enfermería ejecutan con base científica los componentes de la atapa de la evaluación, teniendo que fortalecer todos sus componentes y con respecto al grupo focal se obtuvo como núcleo de sentido que "tienen poco conocimiento y no se aplica la evaluación" lo que se corrobora con expresiones de los participantes como:

PARTICIPANTE 1: "evaluación si.....ya al final de la guardia cuando entregamos la guardia".

PARTICIPANTE 2: "Se observan cambios que se van presentando". 


\section{Conocimiento sobre el proceso de atención de enfermería del profesional en el cuidado que ofrece al paciente: Unidad de cuidados intensivos cardiovascular. Hospital de niños "Dr. Roberto Gilbert Elizalde". 2016-2017}

Vol. 2, núm. Esp., (2018) Irinay Velasco Párraga; Rocío Suárez Fuentes; Geny Rivera Salazar; Herlinda Espinoza Villamar

En base a los resultados de la triangulación coinciden en que "profesionales de enfermería tienen conocimiento científico básico, y no se aplica en la práctica".

La evaluación es una acción continua y formal que está presente a lo largo de todo el Proceso de Atención de Enfermería (P.A.E): es parte integral de cada una de las etapas y se relaciona con la valoración (considerada como una evaluación inicial del sujeto); diagnóstico ( se evalúan los datos para comprobar que la etiqueta diagnostica se ajuste a los mismos); planificación ( cuya fase de priorización se evalúa diagnósticos formulados, percepción del sujeto, recursos de los que se dispone, etc.) y ejecución (se remite a la evaluación de las etapas anteriores).

\section{Conclusiones.}

El proceso de atención de enfermería tiene una base científica, técnica, humanística y ética en cuyas etapas se ejecutan intervenciones de todo tipo para la recuperación de los pacientes y la medición de la calidad del cuidado, esto da como beneficio la interacción con los pacientes, la humanización de la práctica e identidad profesional.

De manera general, en base a los resultados presentados, los profesionales de enfermería de la Unidad de Cuidados Intensivos Cardiovascular que participaron en este estudio, consideran:

"Que el Proceso de Atención de Enfermería mejora el desempeño del profesional, garantizando el cuidado científico, técnico, humanístico y ético, así mismo de manera unánime manifiestan que favorece la planificación, organización del trabajo y es una estrategia para 
Conocimiento sobre el proceso de atención de enfermería del profesional en el cuidado que ofrece al paciente: Unidad de cuidados intensivos cardiovascular. Hospital de niños "Dr. Roberto Gilbert Elizalde". 2016-2017

Vol. 2, núm. Esp., (2018)

Irinay Velasco Párraga; Rocío Suárez Fuentes; Geny Rivera Salazar; Herlinda Espinoza Villamar

optimizar el tiempo, mejorando la calidad del cuidado". Pese a que el Proceso de Atención de Enfermería es considerado importante según el análisis de los resultados, el proceso es asumido de forma superficial debido a su poca aplicación en el desempeño cotidiano por la falta de pertenencia por parte del grupo de profesionales, en lo que se puede comprobar falta de conocimientos científicos-práctico, proceso difícil de ejecutar, la inexistencia de una cultura profesional con respecto al proceso de atención de enfermería en la institución y la falta de registros pertinentes. Los profesionales reconocen: "Que la aplicación del Proceso de Atención de Enfermería no es una práctica consciente y frecuente, y que no se aplican los conocimientos enseñados en su formación en relación al tema expuesto".

En cuanto a la práctica relacionada con las etapas que conforman el Proceso de Atención de Enfermería, los profesionales realizan la valoración con técnicas tradicionales de manera mecánica, guiándose por su experiencia laboral realizándolo de una manera desordenada e incompleta; en cuanto a la realización de diagnóstico de enfermería no se realiza, por lo tanto no existe evidencia de ello. La planificación de las intervenciones se realiza en base a la valoración realizada y al diagnóstico médico, con esto se enfatiza que no se realiza diagnostico enfermero, siendo muy propio e importante para la identidad del profesional de enfermería, las intervenciones que se ejecutan son en base a las ordenes delegadas por los demás integrantes del equipo de salud, las cuales se orientan al cumplimiento de órdenes médicas sin ser registradas.

Además que, como resultados del estudio los profesionales identifican el Proceso de Atención de Enfermería como una identidad profesional que les ayuda a ganar autonomía, sin 


\section{Conocimiento sobre el proceso de atención de enfermería del profesional en el cuidado que ofrece al paciente: Unidad de cuidados intensivos cardiovascular. Hospital de niños "Dr. Roberto Gilbert Elizalde". 2016-2017}

Vol. 2, núm. Esp., (2018) Irinay Velasco Párraga; Rocío Suárez Fuentes; Geny Rivera Salazar; Herlinda Espinoza Villamar embargo no se evidencia en la práctica cotidiana, ya que claramente se demuestra la dependencia de la profesión a la labor médica.

Considerando, los resultados expuestos se enfatiza en que todos los profesionales de enfermería deben ejercer sus funciones a cabalidad, para que adopten un pensamiento crítico, fortaleciendo los conocimientos del proceso para poder aplicarlos en la práctica cotidiana, por lo que se debería incluir en los proyectos educativos, en la investigación y coordinar con las autoridades universitarias para que a través de los internos de enfermería se enfatice la aplicación del proceso de atención de enfermería con cuidado holístico para crear una filosofía de enfermería en el cuidado del paciente, de esta manera incorporarlo institucionalmente.

\section{Recomendaciones.}

Los Profesionales de Enfermería a nivel Institucional deben participar activamente en el modelo de calidad de cuidado de enfermería que se aplica en el hospital, considerando como base científica la "teoría de la resonancia del cuidado" siendo sus componentes: interacción humanizada, buena competencia técnica, investigación permanente del cuidado, trabajo en equipo, buena administración de los servicios y seguridad del paciente. En este modelo el /la enfermero(a) integra su saber, a una realidad concreta o por evidencia y con la puesta en marcha de metodologías y lenguajes propios (taxonomía), y el sistema de registro acorde con las especificidades de la profesión, las situaciones de los pacientes y las condiciones institucionales, en el marco de prestación de los servicios de salud se debe adaptar para lograr la actualización 
Conocimiento sobre el proceso de atención de enfermería del profesional en el cuidado que ofrece al paciente: Unidad de cuidados intensivos cardiovascular. Hospital de niños "Dr. Roberto Gilbert Elizalde". 2016-2017

Vol. 2, núm. Esp., (2018)

Irinay Velasco Párraga; Rocío Suárez Fuentes; Geny Rivera Salazar; Herlinda Espinoza Villamar

permanente de la práctica y su adaptación a los cambios del entorno sociopolítico, económico y cultural.

El departamento de docencia a través de la coordinación de enfermería, debe incluir en su programación cursos de capacitación referente al Proceso de Atención de Enfermería y la utilización de las taxonomías NANDA, NOC, NIC en el desempeño laboral, para ejercer profesionalmente y con eficacia los problemas clínicos-quirúrgicos de los pacientes como estrategia y así poder implementar en todos sus dimensiones el modelo de calidad de cuidado, de esta forma se mantiene la identidad profesional y la atención unificada, para brindar atención humanizada, holística y organizada.

\section{Bibliografía.}

1. Alfaro R. Aplicacion del Proceso Enfermero. Barcelona:; 2003.

2. Diaz Zamora W, Nieto Galvis C. Utilización del Proceso de Enfermería en el Ejercicio Clínico Profesional. Revista de Actualizaciones en Enfermería. 2011 Oct; 3(2).

3. OPS. Política y estrategia regional para la garantía de la calidad de la atención sanitaria, incluyendo la seguridad del paciente. 27.a Conferencia Sanitaria Panamericana. Washington: Organización Panamericana de la Salud; 2007.

4. Rojas JG, Pastor Durango P. Aplicación del proceso de atención de enfermería en cuidados intensivos. Invest Educ Enferm. 2010 Ene; 28(3): p. 323-335.

5. Corzo R, Montes Z, Ramírez M, Reyes R. Conocimiento, importancia y aplicación del método enfermero en la práctica clínica de enfermería. Tesis de licenciatura. México: nstituto Politécnico Nacional, Escuela Superior de Enfermería y Obstetricia; 2004.

6. Chávez T. Barreras que limitan la aplicación del método enfermero en una Institución de Segundo Nivel de Atención. Tesis de licenciatura. México: Instituto Politécnico Nacional, Escuela Superior de Enfermería y Obstetricia; 2006.

7. Smith-Higuchi K, Dulberg C, Duff V. Factors associated with nursing diagnosis utilization in Canada. Nurs Diagn. 1999 Oct; 10(4): p. 137-147.

8. Delaney C, Herr K, Maas M, Specht J. Reliability of nursing diagnoses documented in a computerized nursing information system. Nurs Diagn. 2000 Feb; 11(3): p. 121-134. 


\section{Conocimiento sobre el proceso de atención de enfermería del profesional en el}

cuidado que ofrece al paciente: Unidad de cuidados intensivos cardiovascular. Hospital de niños "Dr. Roberto Gilbert Elizalde". 2016-2017

Vol. 2, núm. Esp., (2018)

Irinay Velasco Párraga; Rocío Suárez Fuentes; Geny Rivera Salazar; Herlinda Espinoza Villamar

9. Björvell C, Wredling R, Thorell-Ekstrand L. Long-term increase in quality of nursing documentation: Effects of a comprehensive intervention. Scand J Caring Sci. 2002; 16(1): p. 34-42.

10. Nahm R, Poston L. Measurements of the effects of an integrated, point-of-care computer system on quality of nursing documentation and patient satisfaction. Computers in Nursing. 2000; 18(5): p. 220-229.

11. González Jurado M. Normalización de la práctica enfermera como contribución a la salud, la calidad asistencial y la seguridad clínica de las personas. Evaluación en los cuidados del paciente neumológico. Tesis. Madrid: Universidad Complutense de Madrid, Facultad de Medicina; 2006.

12. Díaz F, Nieto C. Utilización del proceso de enjfermería en el ejercicio clínico profesional en seis instituciones prestadoras de servicios de salud en Santafé de Bogotá. Tesis (enfermero). Bogota: Universidad Nacional de Colombia; 1998.

13. Gutierrez Lesmes O. Factores que determinan la aplicación del proceso de enfermeria en instituciones hospitalarias de Villavicencio Colombia. Revista Avances de enfermería. 2009 Ene; 27(1): p. 60-68.

14. Sandra Lorena VB, Diana Estefanía VL. APLICACIÓN DEL PROCESO DE ATENCIÓN DE ENFERMERÍA DE LAS ENFERMERAS/OS, QUE LABORAN EN EL HOSPITAL REGIONAL VICENTE CORRAL MOSCOSO, CUENCA 2015. Tesis previa a la obtención del título de Licenciada en Enfermería. Cuenca: Universidad de Cuenca, Escuela de Enfermería; 2015. 